\title{
EXPERIMENTAL STUDY OF FLOW FIELD CHARACTERISTICS IN TIDAL STREAM TURBINE ARRAYS
}

\author{
Martin Nuernberg \\ School of Marine Science and Technology, \\ Newcastle University \\ Newcastle Upon Tyne,NE1 7RU, United Kingdom \\ Email: martin.nuernberg@newcastle.ac.uk
}

\author{
Longbin Tao \\ School of Marine Science and Technology, \\ Newcastle University \\ Newcastle Upon Tyne,NE1 7RU, United Kingdom \\ Email: Longbin.tao@newcastle.ac.uk
}

\section{ABSTRACT}

Tidal currents at many locations around the world have great potential to be used as a large scale renewable energy resource in the future. For large tidal turbine arrays to be commercially viable, the interactions of large devices operating in a confined operating environment need to be understood to optimise the layout of arrays to maximise electricity generation.

This study presents results from a comprehensive experimental investigation of the flow field characteristics within tidal turbine arrays across a number of array layout configurations and current velocities. Up to four small scale turbines were placed in a circulating water channel to investigate the effects of changing array configuration and wake interaction on the flow velocity and turbulence characteristics in small array layouts. Detailed account of the resulting flow field characteristics has been taken by particle image velocimetry measurements at a number of locations within the wake of the array thus providing a large set of instantaneous flow recordings for further analysis of flow features and wake characteristics.

Results are shown for experimental studies of single, three and four turbine arrays and some preliminary comparison between experimental measurements and numerical results are made.

\section{NOMENCLATURE}

ADV Acoustic Doppler Velocimetry

LDV Laser Doppler Velocimetry

PIV Particle Image Velocimetry

HPC High Performance Computing cluster 


\section{INTRODUCTION}

Increasing the share of renewable energy sources in electricity production is a key requirement for achieving goals of reduced fossil fuel dependency and greenhouse gas emissions. With decreasing land area being available for large scale wind energy farms, the oceans as a vast source of sustainable energy have raised significant interest of researchers and technology developers alike. Energy extraction by means of horizontal axis tidal current turbines has seen full scale prototypes deployed at designated testing facilities, supplying electricity to the grid and is currently moving towards demonstrator projects featuring multiple devices.

Numerical simulations ranging from simplified blade element method [1] to fully resolved CFD calculations [2], have been used extensively to investigate and optimise the performance of tidal turbine devices and blade sections. The applicability of various methods of modelling energy extraction from tidal flows that represent actual tidal energy sites more accurately has been investigated through a number of studies. Some attempts have been made to investigate array flow fields using a range of methods to model the tidal turbines in CFD simulations $[3,4]$, however none of these studies are using experimental data alongside the numerical calculations.

Tidal turbines operating in densely spaced arrays of turbines can be optimised in design and location to maximise the electricity generation of the array. Investigating the wake characteristics such as turbulence and velocity recovery, and interactions of multiple wakes within the array will aid in determining optimal spacing parameters for first generation tidal turbine arrays. Due to the relatively shallow waters (approximate water depths of 3 turbine diameters) and confined areas, first generation tidal turbine arrays are likely to experience bounding surface effects resulting from proximity to river or channel banks, free surface and the seabed.

Experimental wake characterisation of single and multiple tidal turbine devices have been presented previously [5-7] utilising actuator disks and scaled three-bladed rotors respectively. Trends in velocity recovery and decay of turbulence have been similar across different experiments, generally reaching a velocity deficit of about $10 \%$ about 20D downstream of the turbines. Differences in wake characteristics are less pronounced with increasing downstream distance showing that, for single devices, ambient flow has large influence on the far wake characteristics while the near wake is dominated by device specific features.

Experimental array studies have investigated the wake characteristics within tidal turbine arrays by means of multiple actuator disks [8] and small three bladed rotors [9, 10]. Wake velocity and turbulence statistics are presented from pointwise ADV and LDV measurements throughout the wake region and show similar trends in velocity recovery up to 20 diameters downstream. Comparison of turbulence characteristics between actuator disks and small bladed rotors by Tedds et al. [7] showed over prediction of turbulent kinetic energy decay up to 9D downstream when applying simplified methods to model tidal turbine devices.

Myers, Bahaj [8] and Stallard et al. [9] observed an optimum lateral distance that gave rise to flow acceleration between two adjacent turbines, hence increasing the available energy to downstream devices. However, it was also noted in [8-10] and that an additional third row of turbines requires increased longitudinal spacing due to increased velocity deficit and turbulence intensities resulting from the two rows of actuator disks installed.

The use of Particle Image Velocimetry (PIV) in tidal turbine experiments is still limited to date however it has been applied across a range of wind turbine wake experiments. Unlike ADV and LDV measurements PIV allows to obtain simultaneous non-intrusive flow field measurements, capturing spatial characteristics of larger coherent turbulence structures than those identified by single-point measurements. 
This study aims to investigate the wake characteristics within a small array configuration of up to 4 turbines across a number of different lateral and longitudinal layout configurations and varying current velocities. PIV measurements are used to investigate the centre-line flow field within and downstream of the array and velocity as well as turbulence statistics are presented herein. Some initial comparison is made between experimental results and preliminary numerical modelling of tidal turbine arrays in OpenFOAM.

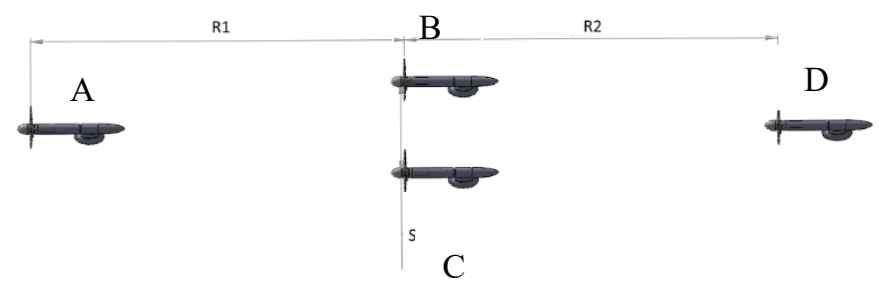

Figure 1 - Array Configuration

\section{FACILITY AND MODEL DESCRIPTION}

The experiments were conducted in the large Circulating-Water-Channel (CWC) at Shanghai Jiao Tong University, Shanghai, China which allows for testing in current velocities ranging from $0.2 \mathrm{~m} / \mathrm{s}$ to $3.0 \mathrm{~m} / \mathrm{s}$ with velocity variations smaller than $\pm 2 \%$, hence representing low ambient turbulence levels. The test section is $8 \mathrm{~m}$ long, $3 \mathrm{~m}$ wide with a water depth of $1.6 \mathrm{~m}$, thus presenting a low vertical and lateral blockage scenario. A glass bottom provides means of sending the vertical laser sheet along the centre-line of the test section with the PIV cameras being located on the side of the tank.

One to four scaled tidal turbine devices have been positioned within the test section on a small support frame that allowed changing the array configuration in terms of lateral and longitudinal spacing (Figure 1). Longitudinal spacing was altered by changing $\mathrm{R} 1$ from $3 \mathrm{D}$ to $5 \mathrm{D}$, while the lateral spacing was changed from $1.5 \mathrm{D}$ to $3 \mathrm{D}$ by increasing the distance (S) between turbine B and C. For presentation of results, the arrays are named according to these distances using ' $\mathrm{L}$ ' for longitudinal and ' $\mathrm{T}$ ' for transverse spacing, hence L3T15 would describe the array with $\mathrm{R} 1=3 \mathrm{D}$ and $\mathrm{S}=1.5 \mathrm{D}$. Current velocities ranging from $0.25 \mathrm{~m} / \mathrm{s}$ to $0.8 \mathrm{~m} / \mathrm{s}$ are used with the model turbine operating at a TSR value of 4 throughout all array tests.

The four identical, bottom supported tidal turbine models have a rotor diameter of $\mathrm{D}=0.28 \mathrm{~m}$ and blades based on the NREL S814 blade section corresponding to a $1: 70^{\text {th }}$ scaled tidal turbine mode. A CAD drawing showing assembled design is given in Figure 3. The models are designed to allow further changes in blade size and configuration, pitch angle and to vary the centre line height of the rotor. In this experiment the rotor centreline was located at $0.35 \mathrm{~m}$ for all scale models deployed. The experimental tidal turbine system utilises Panasonic Minas A5 motors and control unit to record variations in operating conditions of the rotor.

500 PIV recordings were taken at each location with a sampling rate of $4.52 \mathrm{~Hz}$ and processed using LaVision $\mathrm{DaVis}$ 8.2.2. The number of recordings was deemed to be sufficient using moving average of in-stream velocity (x-velocity) and velocity fluctuations (Figure 2).|Results are presented in terms of time-averaged quantities unless otherwise stated. 


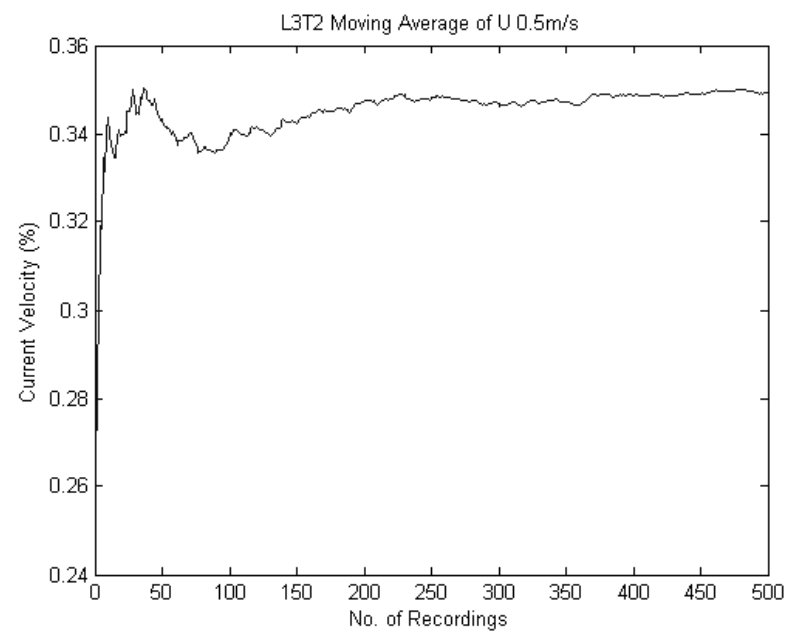

Figure 2 - Moving Average Ux

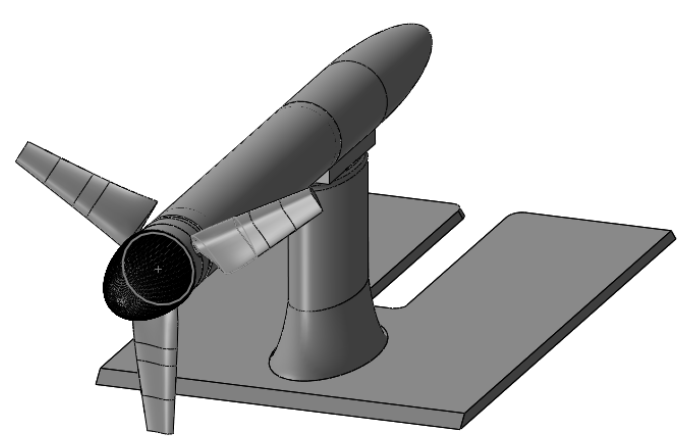

Figure 3 - CAD Model of scaled Model with support plate 


\section{NUMERICAL MODEL}

A numerical CFD model was set up in OpenFOAM to simulate the experiment conditions for more detailed investigation of relevant flow features and further investigations of array layout considerations.

The numerical domain and position of all tidal turbines are re-created from the experiment conditions in the CWC at Shanghai Jiao Tong University. The inlet velocity is set according to experiment conditions and the current direction is from left to right in Figure 4. The domain extends from -5D upstream of the first rotor to 22D downstream, vertically there is a tip-free surface distance of $4 \mathrm{D}$ and the seabed to turbine tip clearance is 0.75D.The CWC free surface is not accounted for at this preliminary stage of simulation, hence top, bottom and the sides of the tank are modelled as walls.

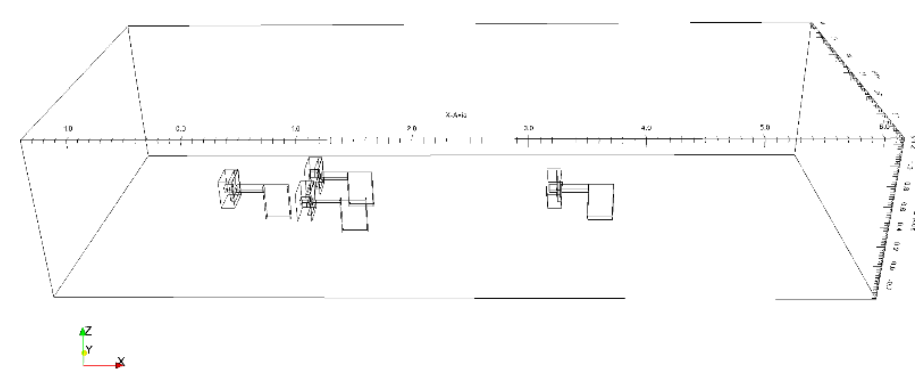

Figure 4 - Arrangement and extent of numerical domain

The simulations are using the arbitrary mesh interface (AMI) capability of OpenFOAM that enables dynamic mesh regions with constant rotation to be modelled in a transient rotor-stator type of approach, shown in Figure 5. Each rotor is set to rotate with a TSR of 4 . The simulations are currently run on in parallel using the PimpleDyMFoam solver with k- $\omega$ SST turbulence model from the OpenFOAM library. A mesh sensitive study investigating the mesh resolution, domain size and boundary conditions is currently being performed on the N8HPC. Depending on experiment configuration and number of turbines being tested, the mesh size varies from 700,000 up to 4.5 million. 


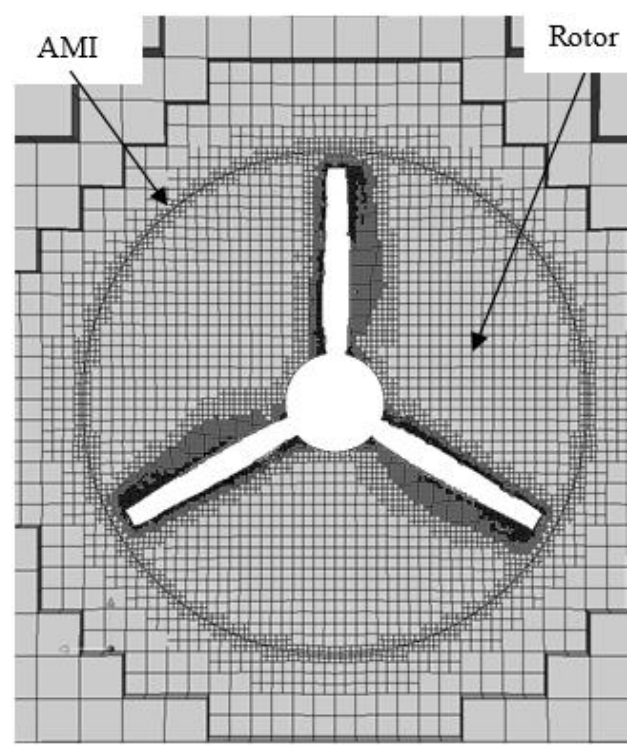

Figure 5 - Rotor-Stator with arbitrary mesh interface

\section{RESULTS AND DISCUSSION}

Wake centre-line measurements in the array at specific locations downstream of a single turbine set-up, using only Turbine A (see Figure 1) show similar trends in velocity recovery with other experimental studies of tidal turbine devices. The wake characteristics are presented in terms of velocity deficit $\left(\mathrm{V}_{\text {def }}=1-\mathrm{U} / \mathrm{U}_{\infty}\right)$, where $\mathrm{U}$ is the recorded velocity and $U_{\infty}$ the ambient current velocity and in-stream turbulence intensity $\left(\mathrm{Ti}=\sigma_{\mathrm{U}} / \mathrm{U}_{\infty}\right)$.

The velocity deficit reduces gradually throughout the wake down to a deficit of less than $10 \%$ of the ambient current velocity from $6 \mathrm{D}$ through to $20 \mathrm{D}$ downstream of the rotor as recorded for single turbine and 3 turbine array shown below in Figure 6. Comparing the velocity recovery between a single turbine and 3 turbine set up, using turbine $A, B, C$ only with $R 1=3 \mathrm{D}$ and $\mathrm{S}=3 \mathrm{D}$, shows a lower initial velocity deficit within the array when 3 turbines are operating, and a lower rate of velocity recovery throughout the wake.

The initial velocity deficit in the centre-line of the array is lower for the 3 turbine case than in the wake of a single turbine. Throughout the array, the rate of velocity recovery between the single turbine and 3 turbine array differ, though the final velocity deficit recorded is very similar for both cases. This lower velocity deficit is likely to be caused by the presence of the second row turbines, forcing more water to flow towards the centre line of the array. The lower rate of wake recovery through the centre part of the array can be explained by the presence of two wakes, either side of the centre wake that limit the amount of mixing occurring with the ambient flow. The decrease in velocity recovery from 15D -17D observed in the 3 turbine case could indicate merging of the slow moving wakes of the second row turbines, followed by a slight increase in wake recovery due to the increased turbulence. 


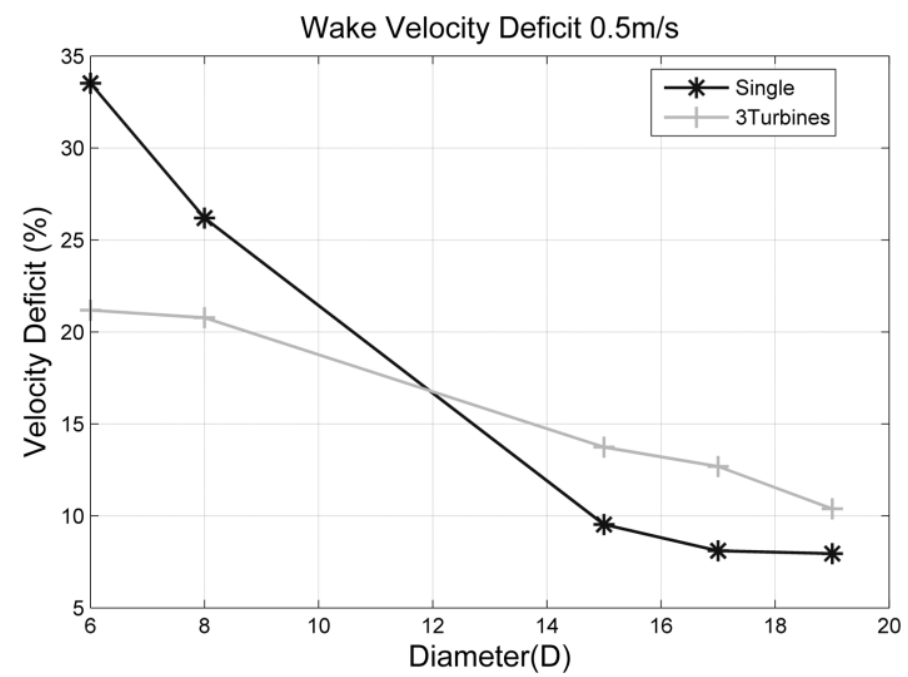

Figure 6 - Velocity deficit comparison in Wake of Single turbine and 3 Turbines at $0.5 \mathrm{~m} / \mathrm{s}$

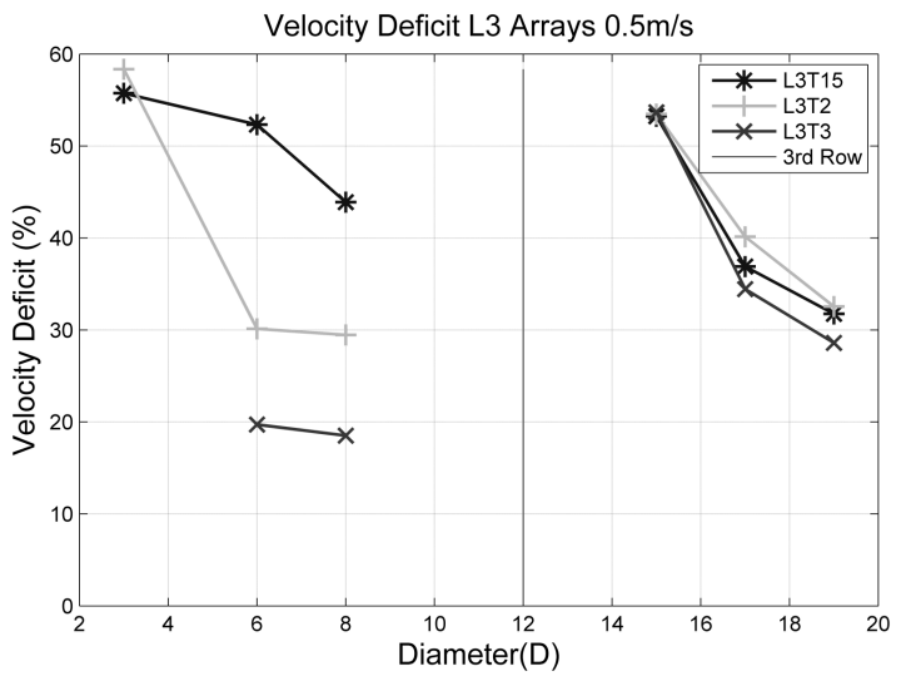

Figure 7 - Comparison of L3 Arrays with $0.5 \mathrm{~m} / \mathrm{s}$ current

A number of different lateral distances between the middle row turbines have been investigated. Results for the velocity deficit are presented for an ambient current velocity of $0.5 \mathrm{~m} / \mathrm{s}$ for the array with R1=3D (Figure 7) and $\mathrm{R} 1=5 \mathrm{D}$ (Figure 8 ). The location of turbine $\mathrm{D}$ on the array centre-line is shown as $3^{\text {rd }}$ row

With increasing spacing between the second row turbines located at 3D downstream of the first turbine, the velocity deficit reduces significantly in the array wake upstream of turbine D (Figure 7). The rate of velocity recovery is very similar between lateral spacing of $2 \mathrm{D}$ and $3 \mathrm{D}$, though showing very slow rate of recovery, while at $1.5 \mathrm{D}$ spacing a faster velocity recovery is observed. This may indicate the influence of close spacing on wake interactions, increasing local turbulence levels, hence speeding up velocity recovery by increased mixing. On the other hand, a slow rate of recovery would suggest low mixing activity with the surrounding flow at the array centre-line, this could mean that the outer wakes have not yet had any impact on the recovery of the centre wake. However, more detailed investigation is needed to support this. 
Downstream of the array, the rate of velocity recovery shows more similarity among all three cases. The velocity deficit for all three cases remains at higher levels, resulting from the last turbine being very much located in the wake of the upstream turbines. A faster rate of recovery in this area does indicate increased levels of turbulence that aid in re-energising the wake, hence reducing the velocity deficit.

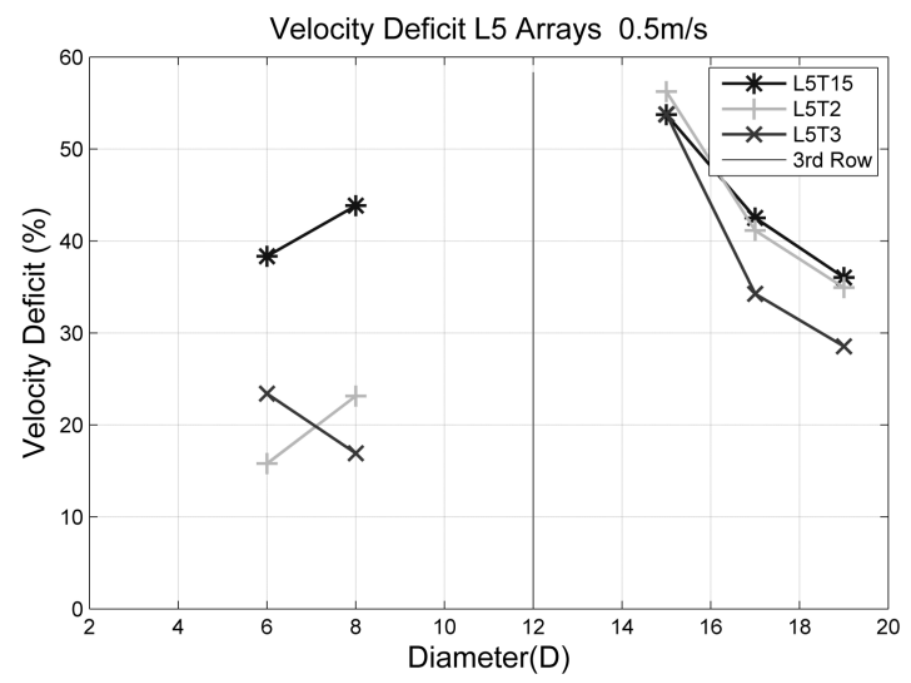

Figure 8 - Comparison of L5 Arrays with $0.5 \mathrm{~m} / \mathrm{s}$ current

The wake characteristics for array configuration with $\mathrm{R} 1=5 \mathrm{D}$, presented in Figure 8, allow for comparison of less data points due to the presence of model structures in the recording window which led to some spurious results in between the two middle row turbines. Some further investigation is needed to determine whether the increase in velocity deficit seen between $6 \mathrm{D}$ and $8 \mathrm{D}$ is due to wake interaction with the closer spaced middle row wakes or a result of processing PIV images. Comparing the velocity deficit within the array, at $8 \mathrm{D}$ for all three lateral spacing, the velocity deficits are lower than the previous array

Velocity recovery downstream of the array shows very similar behaviour to the previous array, showing little influence of the longitudinal spacing of the upstream turbines on the wake behaviour downstream. Velocity deficits are slightly higher due to being located closer to the upstream turbine (7D compared to 9D).

The deficit remains highest for the closest spacing with longitudinal distance of 5D between the first two rows, however, for 3D separation the velocity deficit is highest downstream of the array for the 2D lateral spacing. This may indicate some flow acceleration occurring between the two turbines that reduces the velocity deficit slightly stronger.

Figure 9 shows a comparison between the turbulence intensity in the wake of two array configurations. It can clearly be seen that with reduced spacing of $1.5 \mathrm{D}$ of the middle row turbines, the turbulence intensity at the centre line of the array is increased and persists at higher levels further downstream. This is in good agreement with the increased rate of velocity recovery, especially between 7D -9D downstream of turbine A, shown above (Figure 7). 

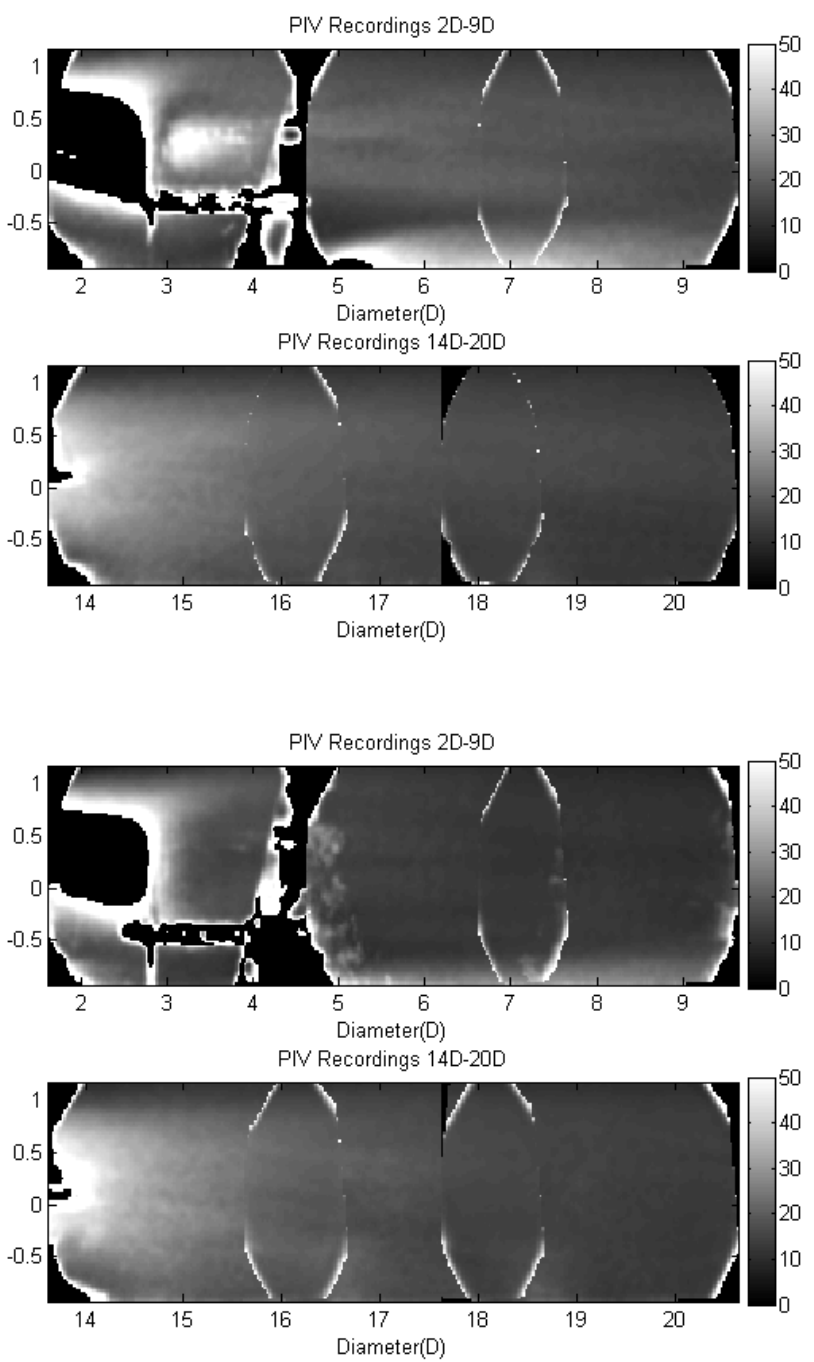

Figure 9 - Turbulence Intensity (\%) comparison of L3T15 (top) and L3T2 (bottom)

Comparing the turbulence intensity characteristics of all array configurations, Figure 10, shows higher Ti in the middle part of the array when the longitudinal spacing R1=5D. For both longitudinal spacings of 3D and 5D, it can be seen that turbulence levels decay faster downstream of the last turbine, however the turbulence remains at slightly higher levels due to the increased turbulence levels for the inflow to turbine D compared to turbine A at the front line of the array. 

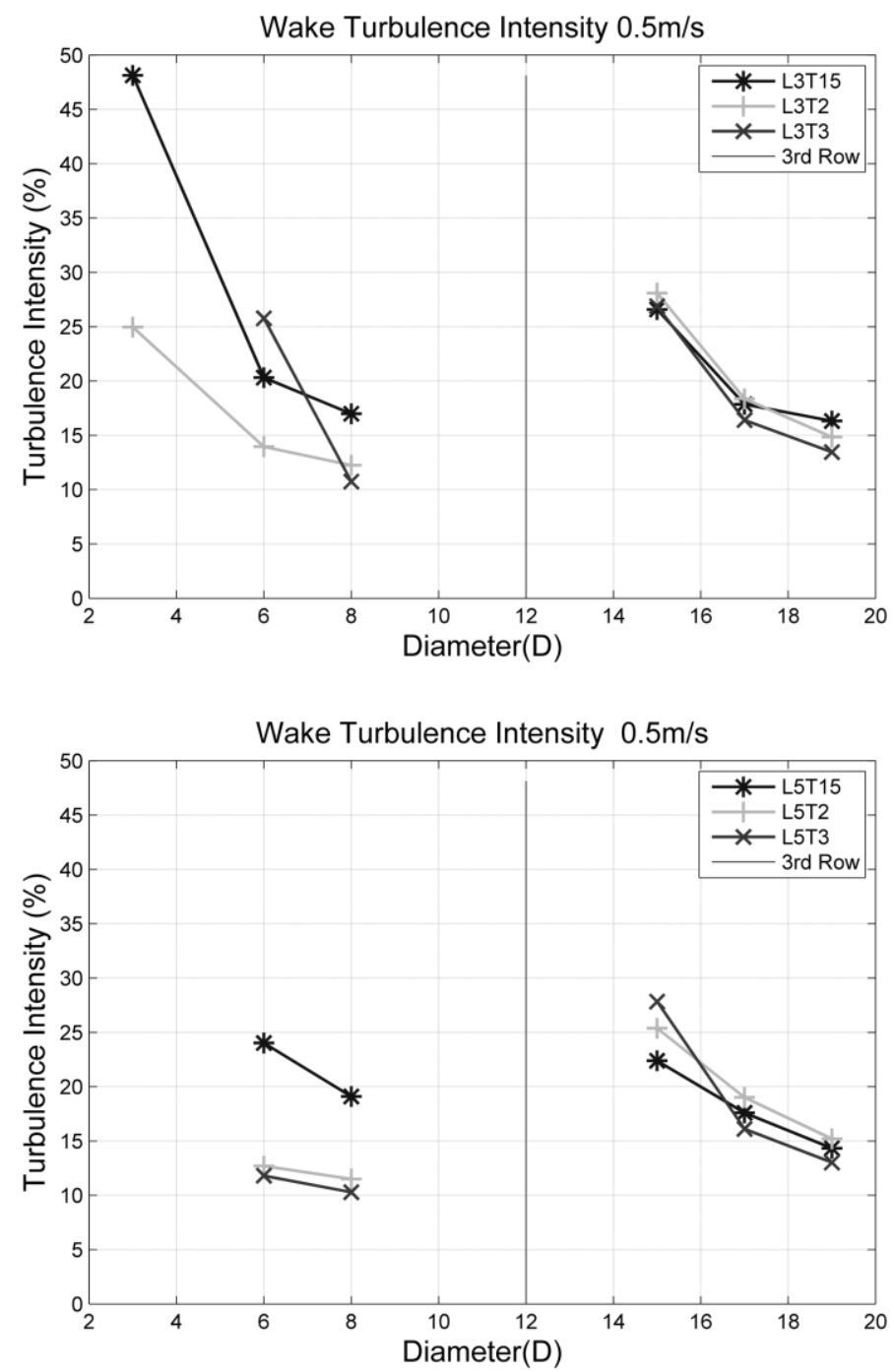

Figure 10 - Comparison of Turbulence Intensity characteristics in arrays with 3D longitudinal spacing (Top) and 5D (Bottom) 
Wake Turbulence Intensity $0.5 \mathrm{~m} / \mathrm{s}$

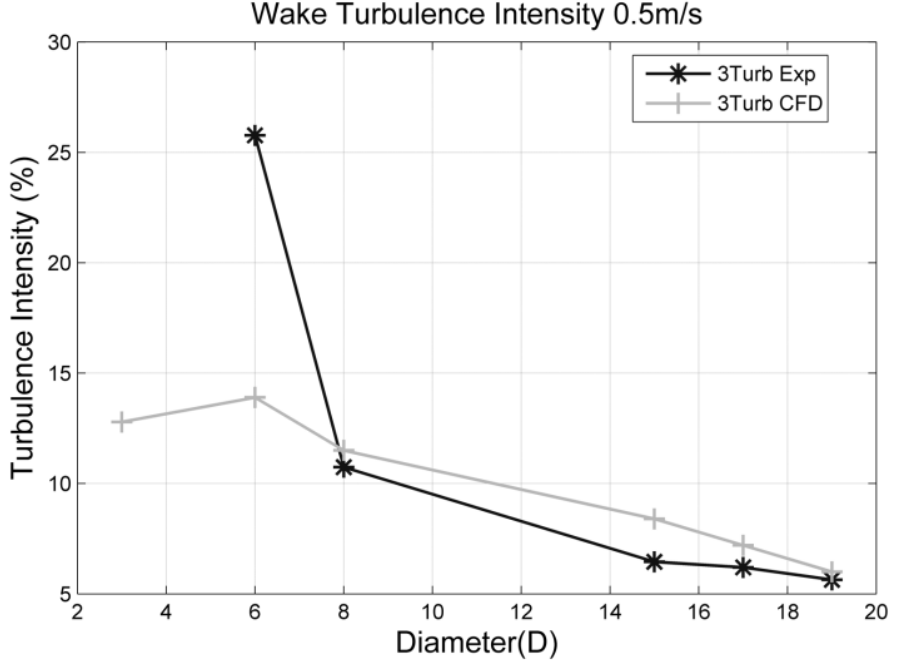

Figure 11 - Preliminary comparison between CFD and experiment Results (3Turbine, 0.5m/s)

Preliminary comparison between CFD and Experiment results in Figure 11 shows reasonable agreement in terms of turbulence intensity. The velocity deficit presented in Figure 12 does not agree well in terms of deficit at specific locations within the wake, however the trend of velocity recovery, especially for the far wake agrees quite well with that of the experiments. The preliminary comparison is based on time averaged data of a much shorter period, which would affect the data extracted from the near wake region more than the far wake region where the flow is less turbulent.

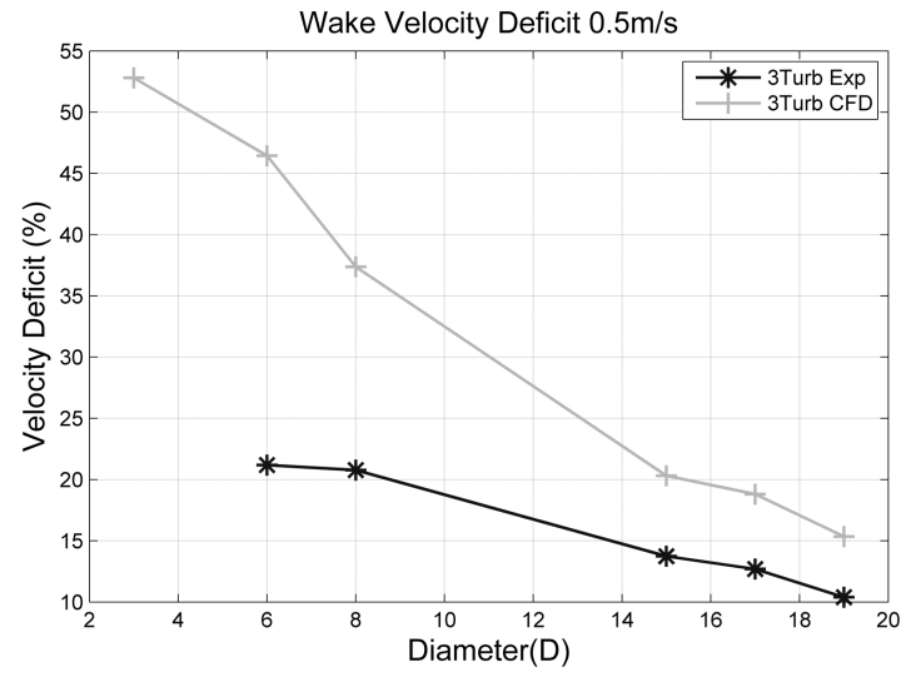

Figure 12 - Preliminary comparison between CFD and experiment results (3 Turbines, 0.5m/s) 


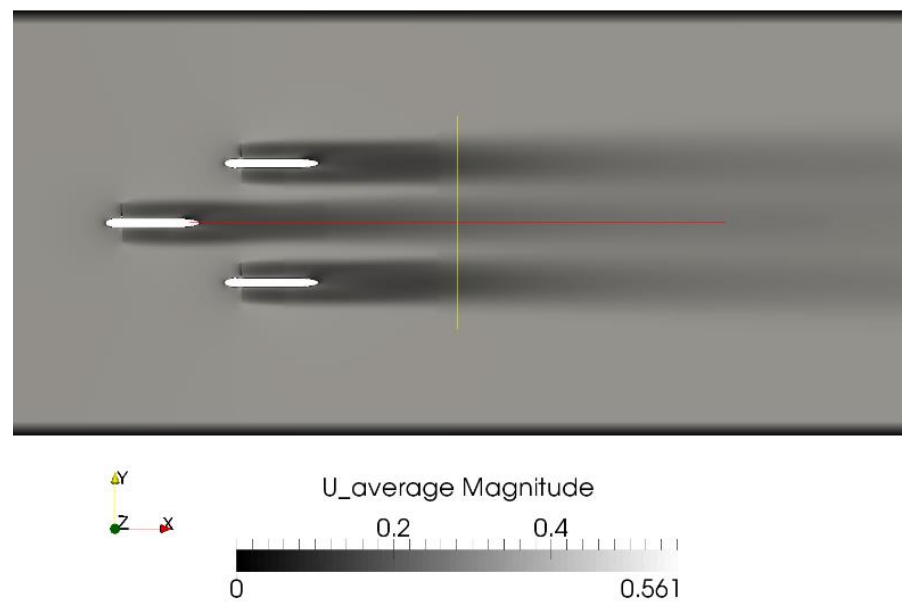

Figure 13 - Wake at hub height from CFD calculations

Regarding beneficial flow effects from locating turbines in close lateral separation, as can be seen in Figure 13, there are areas between the centre turbine and side turbine wakes that experience some flow acceleration due to blockage effects that aid in re-energising the wake and reducing the velocity deficit of the centre wake in Figure 14, showing the lateral velocity profile at hub height at two different positions. This would allow a closer longitudinal spacing for an array, thus increasing the energy density of commercial arrays.

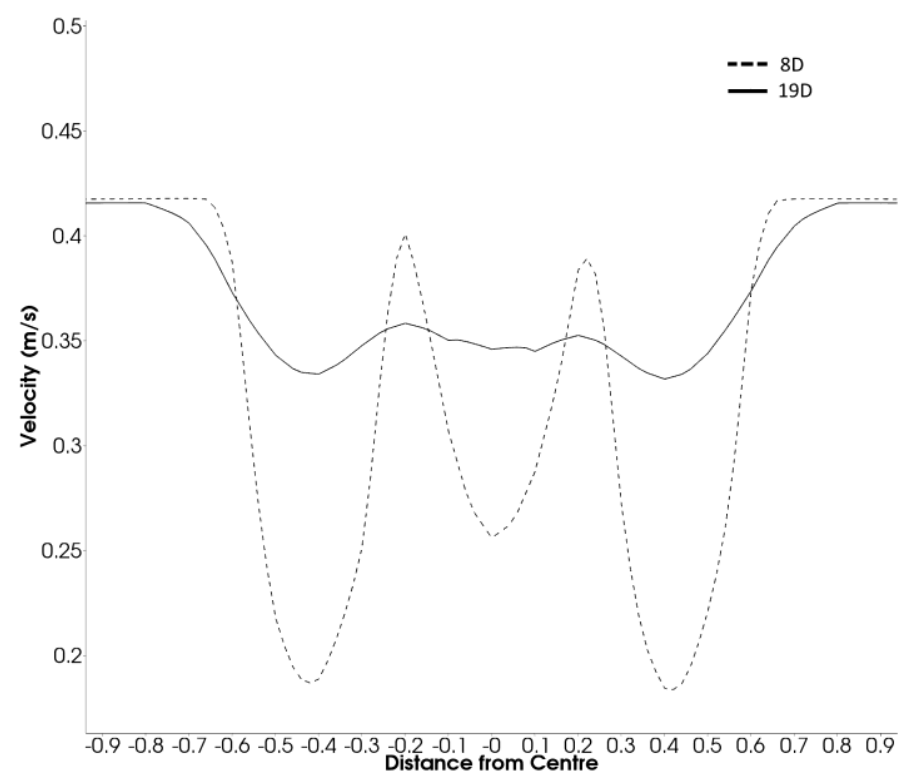

Figure 14 - Velocity Profiles of array wake for 3 Turbines 


\section{CONCLUSION}

Wake velocity and turbulence characteristics have been recorded for a range of scale mode tidal turbine array configurations tested in a circulating water channel.

Results suggest, for a low ambient turbulence environment, that longitudinal spacing in a staggered array configuration has a small effect on the wake recovery in terms of velocity deficit and turbulence intensity. The lateral spacing of the middle row turbines causes more significant variations due to a shift in location where wakes of the turbines reach the array centre-line. Some of the results point towards flow acceleration occurring at closer spacing that reduce initial wake velocity deficit within the staggered set-up.

Preliminary numerical simulation of experiments in OpenFOAM are currently being performed and show some good agreement in turbulence characteristics, however the velocity data in the near wake region of turbine A shows significant differences between experiment and numerical modelling. Close lateral spacing aids in recovering velocity quicker in the centre wake than the two outer wakes.

Further improvements of the numerical simulation to more accurately represent the near wake flow field are currently being carried out implementing the results of mesh sensitivity and improved boundary conditions. The final array computations will then be used for comparison of different arrays settings in terms of flow field, as well as estimated generated power by different arrays using the CFD calculations.

\section{ACKNOWLEDGMENTS}

The authors gratefully acknowledge the support received in conducting this experiment by the School of Naval Architecture, Ocean and Civil Engineering at Shanghai Jiao Tong University and the British Council (China) under the "Sino-UK Higher Education Research Partnership" as well as LaVision for providing support and software for the PIV analysis. The numerical work made use of the facilities of N8 HPC provided and funded by the N8 consortium and EPSRC (Grant No.EP/K000225/1). The center is co-ordinated by the Universities of Leeds and Manchester. 


\section{REFERENCES}

1. Bahaj, A.S., W.M.J. Batten, and G. McCann, Experimental verifications of numerical predictions for the hydrodynamic performance of horizontal axis marine current turbines. Renewable Energy, 2007. 32(15): p. 2479-2490.

2. McNaughton, J., et al., A simple sliding-mesh interface procedure and its application to the CFD simulation of a tidal-stream turbine. International Journal for Numerical Methods in Fluids, 2014. 74(4): p. 250-269.

3. Lee, S.H., et al., A numerical study for the optimal arrangement of ocean current turbine generators in the ocean current power parks. Current Applied Physics, 2010. 10(2, Supplement): p. S137-S141.

4. Churchfield, M.J., Y. Li, and P.J. Moriarty, A large-eddy simulation study of wake propagation and power production in an array of tidal-current turbines. Philos Trans A Math Phys Eng Sci, 2013. 371(1985): p. 20120421.

5. Myers, L.E. and A.S. Bahaj, Experimental analysis of the flow field around horizontal axis tidal turbines by use of scale mesh disk rotor simulators. Ocean Engineering, 2010. 37(2-3): p. 218-227.

6. Mycek, P., et al., Experimental study of the turbulence intensity effects on marine current turbines behaviour. Part I: One single turbine. Renewable Energy, 2014. 66(0): p. 729-746.

7. Tedds, S.C., I. Owen, and R.J. Poole, Near-wake characteristics of a model horizontal axis tidal stream turbine. Renewable Energy, 2014. 63(0): p. 222-235.

8. Myers, L.E. and A.S. Bahaj, An experimental investigation simulating flow effects in first generation marine current energy converter arrays. Renewable Energy, 2012. 37(1): p. 28-36.

9. Stallard, T., et al., Interactions between tidal turbine wakes: experimental study of a group of three-bladed rotors. Philos Trans A Math Phys Eng Sci, 2013. 371(1985): p. 20120159.

10. Mycek, P., et al., Experimental study of the turbulence intensity effects on marine current turbines behaviour. Part II: Two interacting turbines. Renewable Energy, 2014. 68: p. 876-892. 